MKG-Chirurg 2021 · 14:71-73

https://doi.org/10.1007/s12285-021-00292-8

Angenommen: 16. Februar 2021

(c) Springer Medizin Verlag $\mathrm{GmbH}$, ein Teil von Springer Nature 2021

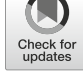

\title{
D. Groß
}

Institut für Geschichte, Theorie und Ethik der Medizin, Medizinische Fakultät, Universitätsklinikum Aachen, RWTH Aachen University, Aachen, Deutschland

\section{Pioniere der MKG-Chirurgie und ihre (fach)politische Rolle im "Dritten Reich"}

brachten. Andererseits erscheint es aber auch nicht zulässig, das Leben einer Person allein an ihrer politischen Haltung bzw. ihrer Belastung im Nationalsozialismus $\mathrm{zu}$ bemessen und dabei erzielte fachliche Leistungen zu ignorieren - ein Vorwurf, der „NS-Forschern“ verschiedentlich gemacht wird.

Eine enge biographische Verknüpfung fachlich-wissenschaftlicher Gesichtspunkte mit dem soziopolitischen Kontext erscheint aber auch noch aus einem weiteren Grund lohnend: Das „Dritte Reich“ war gerade für das junge Fach Kieferchirurgie eine Zeitphase, in der große Hoffnungen, aber auch enorme berufspolitische Unsicherheiten bestanden: Die Hoffnungen fußten auf der im Ersten Weltkrieg gewonnenen Erkenntnis, dass das Fach angesichts zahlloser Kiefer- und Gesichtsverletzten eine zentrale Rolle in der Kriegschirurgie - und damit in der medizinischen und gesellschaftlichen Bewältigung der Kriegsfolgen - spielte. Gerade im „Experimentierfeld“ Krieg gelangen etliche fachliche und operative Neuerungen, die den verletzten Soldaten und Zivilisten neue Lebensperspektiven eröffneten. Sie machten die aufkeimende Spezialdisziplin zu einem „kriegswichtigen“ Fach, dem Politik und Gesellschaft in der Zwischenkriegszeit und v.a. seit dem Beginn des Zweiten Weltkrieges (1939) hohe Bedeutung beimaßen. Die Unsicherheiten erwuchsen ihrerseits aus der Tatsache, dass der Aus- und Weiterbildungsweg zum damaligen „Kieferchirurgen“ in der Ära des Nationalsozialismus (noch) ausgesprochen heterogen war. Diese Heterogenität betraf die formale
Qualifikation der führenden zeitgenössischen Fachvertreter, die bestehenden rechtlichen Bestimmungen und die fachinternen Meinungen in der „Aus- und Weiterbildungsfrage“.

\section{Disparate formale Qualifikation}

In den 1930er und 1940er Jahren gab es neben doppelt approbierten Kieferchirurgen, wie z. B. Georg Axhausen und Martin Waßmund, auch Fachvertreter, die eine rein zahnärztliche Ausbildung absolviert hatten, aber dennoch - meist aufgrund umfangreicher kriegschirurgischer Erfahrungen - in der MKG-Chirurgie tätig wurden und dort breite Anerkennung erlangten. Beispiele für diese zweite Gruppierung sind Christian Bruhn oder Franz Ernst. Zum Dritten fanden sich reine Chirurgen, die sich auf die Kiefer- bzw. Gesichtschirurgie spezialisierten und ebenfalls sehr respektiert wurden, wie etwa Erich Lexer oder Rudolf Klapp. Mit dem „Anschluss“ Österreichs an das Deutsche Reich kam 1938 noch eine vierte Gruppierung hinzu: Nun waren auch die Wiener und Grazer Kieferchirurgen $\mathrm{zu}$ „Reichsdeutschen“ geworden. In Österreich gab es gar kein grundständiges Studium der Zahnheilkunde. Die Betreffenden hatten dementsprechend ein Medizinstudium durchlaufen und sich danach postgradual in der Zahn-, Mund- und Kiefer(ZMK)-Heilkunde und der Kieferchirurgie weitergebildet. Prominente Beispiele für diesen Typus waren Hans Pichler, Otto Hofer oder Hermann Wolf. verfolgten und auf diese Weise so manche undifferenzierte „Hagiographie“ hervor- 


\section{Widersprüchliche gesetzliche Bestimmungen}

Die skizzierte Heterogenität wurde im „Dritten Reich“ durch die rechtlichen Bestimmungen noch verschärft: So führte der Gesetzgeber neben dem bereits 1924 etablierten „Facharzt für Zahn-, Mund- und Kieferkrankheiten“ für Doppelapprobierte im Jahr 1935 einen „Fachzahnarzt für Kieferchirurgie" für rein zahnärztlich Approbierte ein. Als Letzterer dann 1942 auch noch in „Fachzahnarzt für Kieferkrankheiten“ umbenannt wurde, war ein Höchstmaß an Verwirrung erreicht.

\section{Fachinterne Diskurse}

Vor diesem Hintergrund nimmt es nicht wunder, dass unter den Kieferchirurgen in der Zwischenkriegszeit eine Diskussion über den künftigen Ausbildungsgang entbrannt war, die letztlich bis in die 1950er Jahre anhalten sollte. Eine wichtige Stimme in diesem Diskurs war Georg Axhausen. Er war eng mit der 1932 gegründeten „AG Kieferchirurgie“ (heute: AGOKi) verbunden, die deshalb auch „Axhausen-Gesellschaft" genannt wurde. Er vertrat die Auffassung, dass das Fachgebiet „seinem Wesen nach zur Zahnheilkunde“ gehöre. Daher propagierte er primär ein Studium der Zahnheilkunde und eine zusätzliche "gediegene chirurgische Vorbildung"; die ärztliche Approbation hielt er für ,wünschenswert, aber nicht für unerlässlich“. Dementsprechend stand die AG Kieferchirurgie auch rein zahnärztlich Approbierten offen; sie war und blieb bewusst unter dem Dach der zahnärztlich geprägten DGZMK angesiedelt.

Eine Gegenposition vertrat etwa Martin Waßmund: Er initiierte 1951 die DGKG (heute: DGMKG) als eine Organisation, die explizit Doppelapprobierten vorbehalten war und unabhängig von der DGZMK bestehen sollte - worin Axhausen wiederum eine gefährliche Abgrenzung zur Zahnheilkunde zu erkennen glaubte.

Diese Ausführungen machen deutlich, dass die zeitgenössischen Kieferchirurgen in der Ära des „Dritten Reiches“ letztlich drei Herausforderungen gegenüberstanden: Auf der wissenschaftlichen und operativen Ebene galt es, das Fach weiterzuentwickeln und zugleich für künftige fachliche Herausforderungen - wie z.B. Kriegssituationen - zu wappnen, auf der persönlichen Ebene ging es darum, sich gegenüber der NSIdeologie und zur NS-,Gesundheitspolitik“ zu positionieren (und das eigene Handeln nach dieser Position auszurichten), und auf der berufspolitischen Ebene war es erforderlich, sich mit der virulenten "Aus- und Weiterbildungsfrage“ auseinanderzusetzen, um ein stark heterogenes Tätigkeitsfeld zu einem homogenen, zukunftsfähigen Spezialfach weiterzuentwickeln.

An ebendieser Stelle setzt unsere Serie an: Sie beleuchtet die wissenschaftshistorisch bedeutendsten Fachvertreter in der Ära des „Dritten Reichs“ und stellt hierbei die Fragen nach ihrem jeweiligen wissenschaftlichen und klinischen Stellenwert, ihrer (partei)politischen Haltungen und Aktivitäten, aber auch nach ihrer Position und Rolle in der „Aus- und Weiterbildungsfrage“.

Wir hoffen, dass Ihnen die Reihe gerade durch diese historische Betrachtungsweise einen neuen Blick auf Ihre Fachdisziplin eröffnet - getreu dem Motto: (Nur) wer die Geschichte kennt, kann die Zukunft gestalten ...

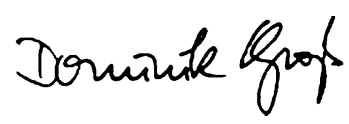

Prof. Dr. med. Dr. med. dent. Dr. phil. Dominik Groß

\section{Korrespondenzadresse}

Prof. Dr. Dr. Dr. D. Groß
Institut für Geschichte,
Theorie und Ethik der
Medizin, Medizinische
Fakultät, Universitätsklinikum
Aachen, RWTH Aachen
University
Wendlingweg 2, Gebäude
MTI 2, 52074 Aachen,
Deutschland
dgross@ukaachen.de

Interessenkonflikt. D. Groß gibt an, dass kein Interessenkonflikt besteht.
Elmar Ludolph

Ärztliche Begutachtung von $A$ - Z

Fachbegriffe, die der ärztliche Gutachter kennen muss

Berlin Heidelberg: Springer-Verlag 2020, 2. Auflage, 391 S., (ISBN: 978-3662-61739-7), Softcover 24,99 EUR

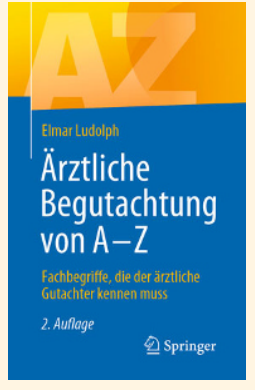

Wussten Sie, dass eine "funktionelle" Verletzung ein Paradoxon ist, da durch die Verletzung nicht nur die Funktion gestört wird sondern in der Organmedizin mit ihr auch ein

entsprechendes morphologisches Substrat verbunden ist? Oder wussten Sie, dass die Wortbedeutung „Unfall“ in den verschiedenen Rechtsgebieten ganz unterschiedlich ausgefüllt ist? Dieses Werk ist nicht einfach ein Wörterbuch, sondern man erhält Antworten auf obige und viele weitere Fragen.

Das Buch ist ein unabdingbares Nachschlagewerk in jeder Bibliothek eines gutachtlich tätigen Arztes, denn sowohl dem Youngster als auch dem gutachtlichen Fuchs sei ans Herz gelegt, immer wieder einmal die von ihm benutzten Bezeichnungen zu hinterfragen und den Sinninhalt nachzuschlagen. Aber auch dem Juristen, der sich mit versicherungsrechtlichen Fragen beschäftigt, sei dieses Buch wärmstens empfohlen, es trägt zu einem gegenseitigen Verständnis zumindest im Sprachgebrauch bei, da die erläuterten Begriffe die entscheidenden Berührungspunkte von Recht und Medizin sind.

Das Buch kann dabei zwar keinen Anspruch auf Vollständigkeit aller Begriffe und Bezeichnungen erheben, in der nunmehr vorliegenden 2. Auflage wurden aber neue Begriffe aufgenommen, zentrale überdacht und umformuliert und sie orientiert sich an der jeweils gültigen Rechtsprechung.

H.-T. Klemm (Bayreuth/Erlangen) 
Hier steht eine Anzeige.

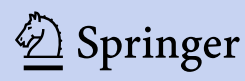

